\section{ALGINATE-MEDIATED SYNTHESIS OF HETERO-SHAPED SILVER NANOPARTICLES AND THEIR HYDROGEN- PEROXIDE SENSING ABILITY}

*Sneha Bhagyaraj, *Igor Krupa

${ }^{a}$ Center for Advanced Materials, Qatar University, P.O. Box 2713, Doha, Qatar.
Faculty and PostDoc, Science and Engineering

\section{Introduction}

Silver nanoparticles have been the focus of extensive research for many decades due to their unique physical, chemical and electrical properties, which make them suitable for diverse applications. Among the synthesis techniques for silver nanoparticles, green synthesis protocols, which can be used to prepare stable and unique nanostructures, are gaining more attention. Naturally derived materials such as biopolymers could be a good alternative to toxic reagents such as trisodium citrate and borohydrides for use in synthesis methods due to the absence of biohazards associated with their byproducts and subsequent waste management.

Alginate is a naturally occurring polysaccharide composed of linear polymer chains of $\alpha$-L-guluronate $(G)$ and $\beta$-d-mannuronate (M) units in such an arrangement that the copolymer units appear to be in the form of an irregular block pattern. The biodegradability and biocompatibility of alginate, together with its ability to form gels upon reacting with divalent cations, make it highly useful in various biological applications, such as developing 3D tissue/organs for tissue engineering, wound healing scaffolds, and drug delivery platforms.

Herein, we report a new, green and facile route for synthesizing Ag-NPs having heterostructured morphologies using alginate and glucose as the capping and reducing agents, respectively. The ascapping and reducing agents, respectively. The assensing activity towards the presence of hydrogen peroxide even at a very low concentration of 10-7 M.

\section{Experimental Methods}

Method: For the reaction, to $100 \mathrm{~mL}$ of distilled water taken in a round bottom flask was added $1.0 \mathrm{~g}$ of sodium alginate powder, and the resulting solution was heated to $60{ }^{\circ} \mathrm{C}$ with vigorous stirring to obtain a clear solution. After $60 \mathrm{~min}, 5 \mathrm{~mL}$ of AgNO3 solution (1 M) was added to the alginate solution with continuous stirring to obtain $\mathrm{Ag}+/$ alginate solution. This step was followed by the addition of $10 \mathrm{~mL}$ of glucose solution $(0.08 \mathrm{M})$ under continuous stirring. The reaction was maintained at $80^{\circ} \mathrm{C}$ and run for 48 hours. Aliquots were taken at different time intervals $(1,5,24 \& 48$ hours) to monitor the grew of intervals $(1,5,24$ particles. To analyze the sensing properties of the Ag-NPs, different concentrations of hydrogen peroxide solutions $(2000 \mu \mathrm{L})$ were added to the silver nanoparticle solution in a quartz cuvette at a ratio of 1:1.5. The change in the UVvis spectrum with varying concentrations of $\mathrm{H} 2 \mathrm{O} 2$ in the range from 10-1 to 10-7 due to the catalytic reaction between the silver nanoparticles and hydrogen peroxide was monitored.

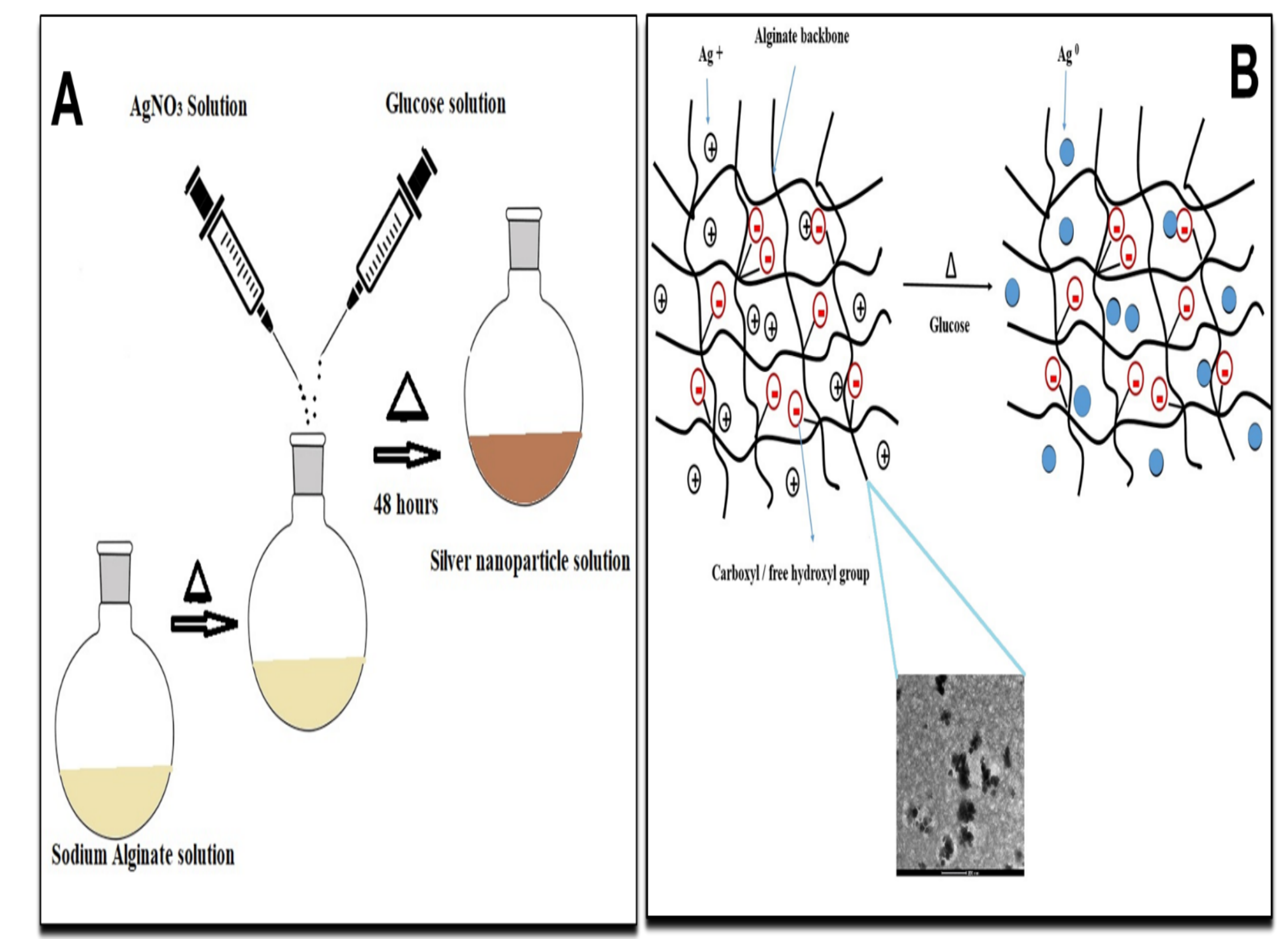

Figure 1: (A) Schematic representation of the synthesis of sodium alginate-capped Ag-NPs (B) Schematic representation of the formation of alginate-capped Ag-NPs

\section{Results and discussion}

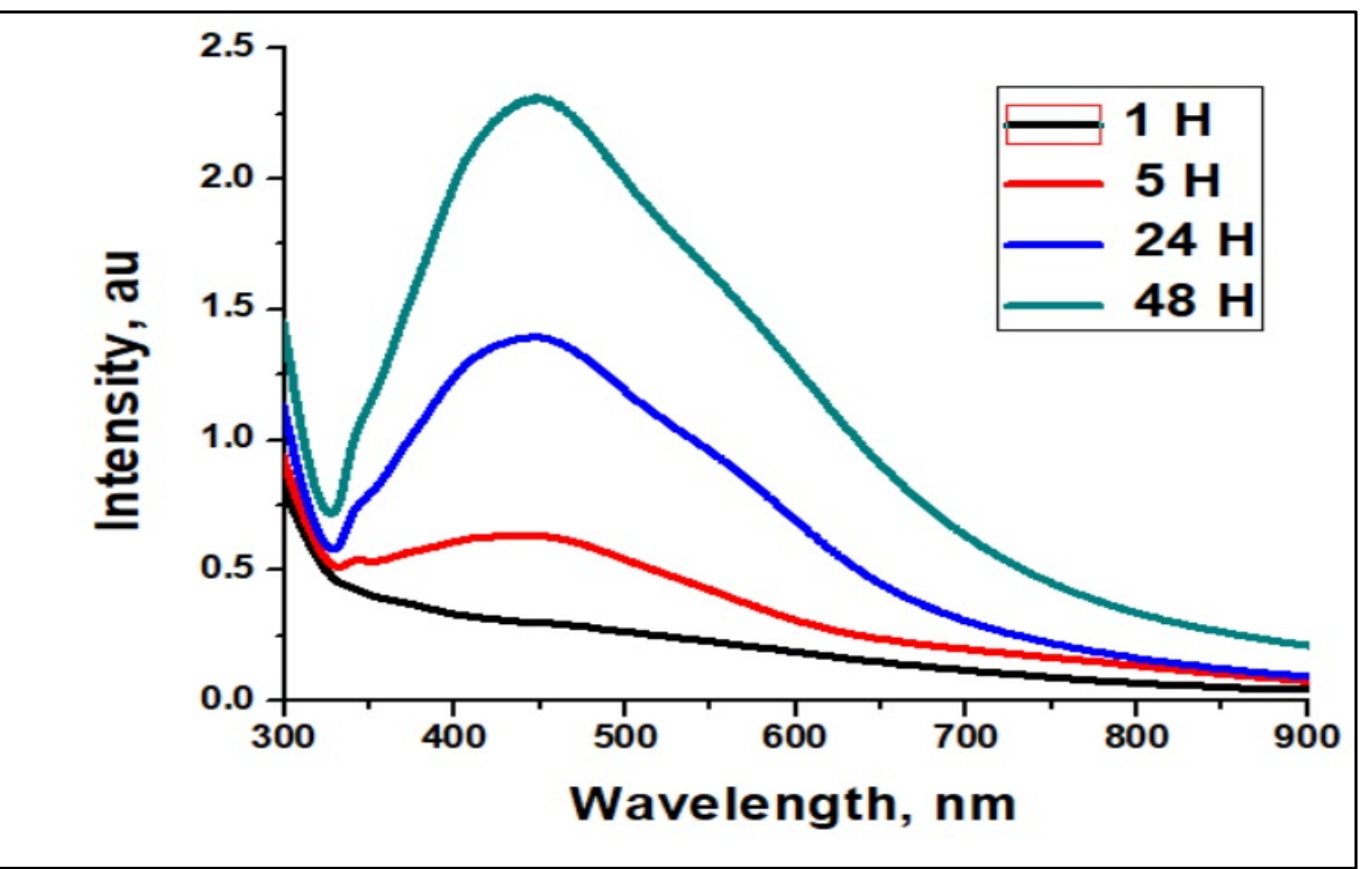

Figure 2: (A) UV absorption spectra of alginatecapped Ag-NPs at different reaction times.

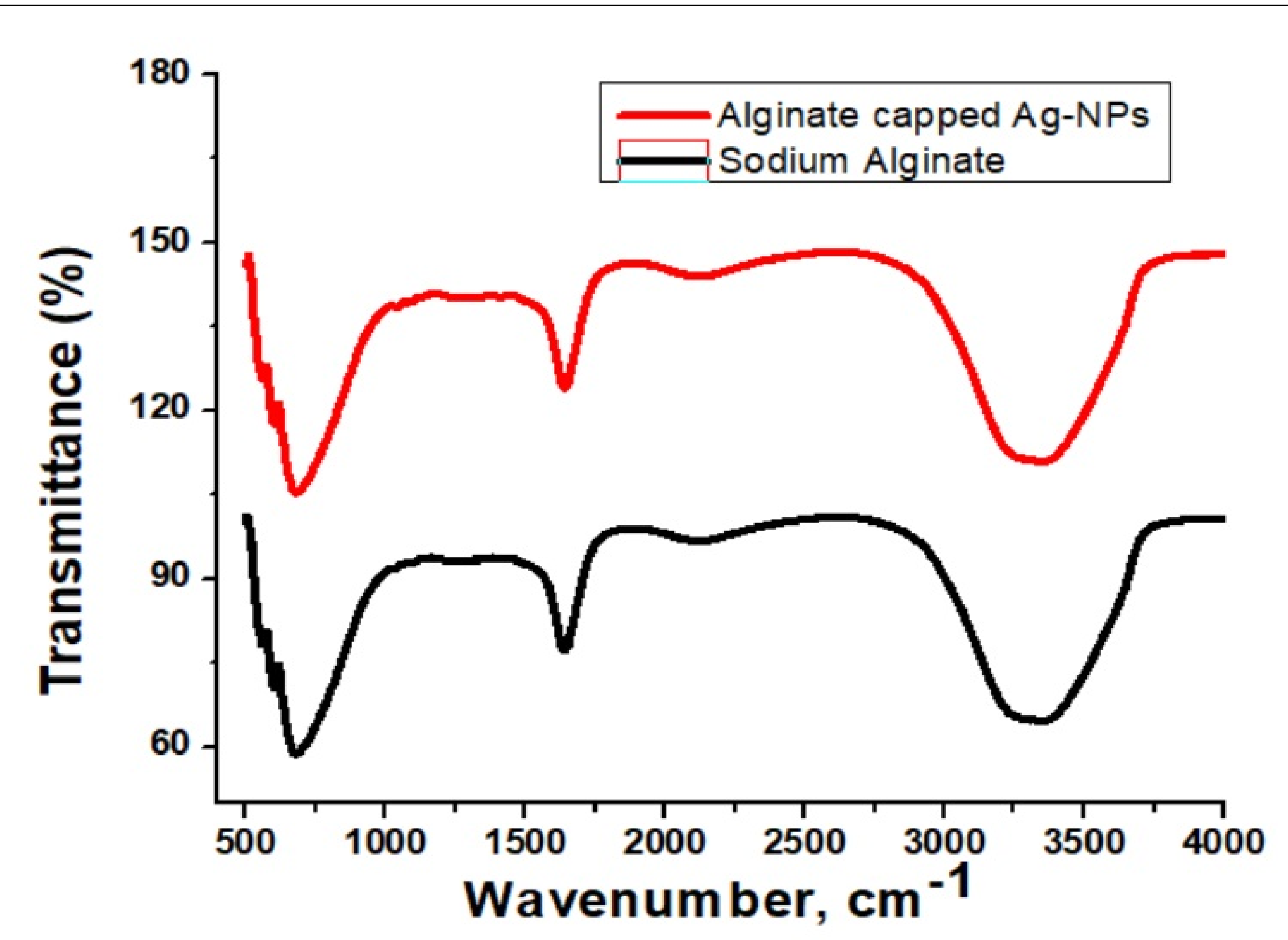

Figure 3: FTIR spectra of the Ag-NPs.

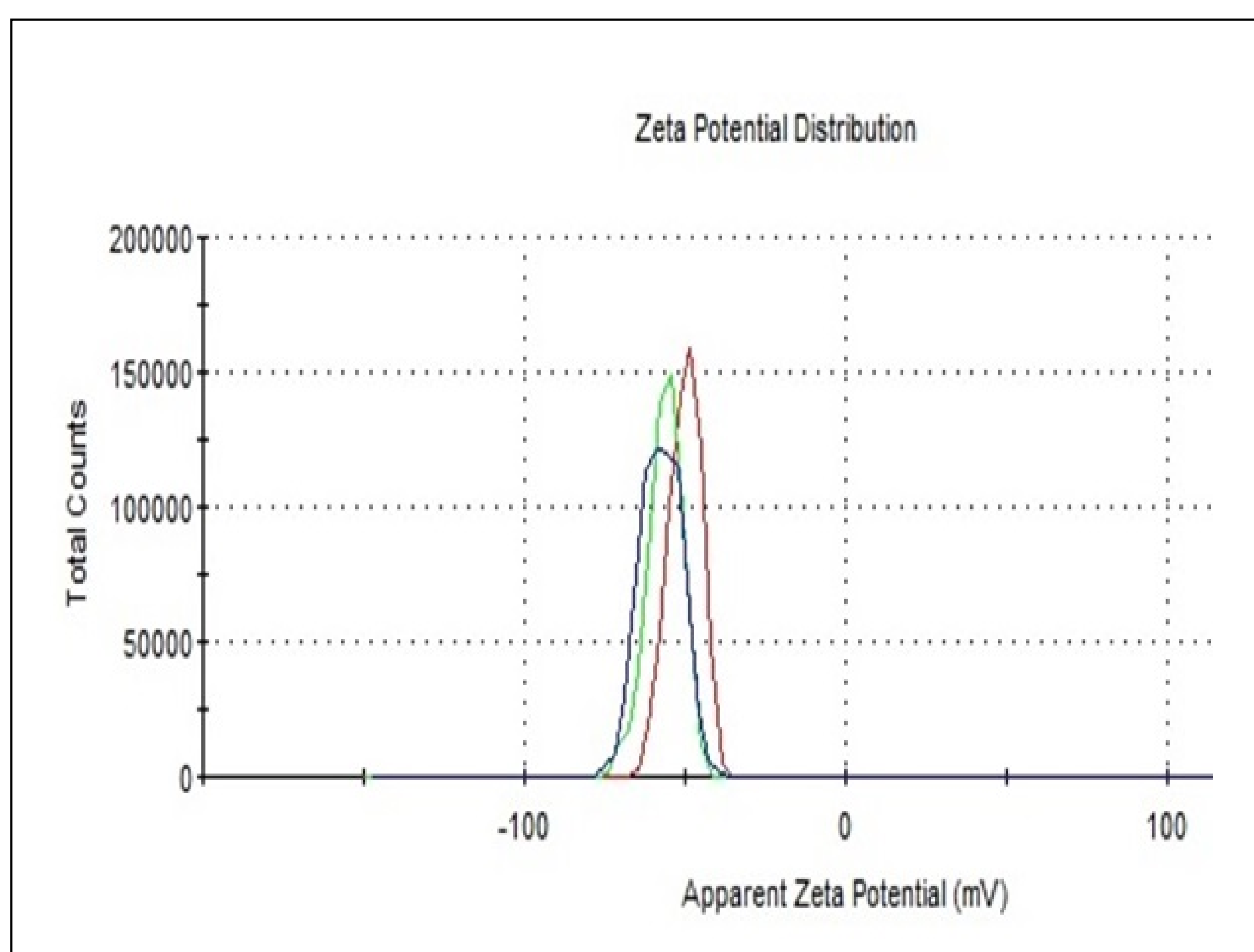

Figure 6: Zeta potential distribution of Ag-NPs at $48 \mathrm{~h}$ reaction time.

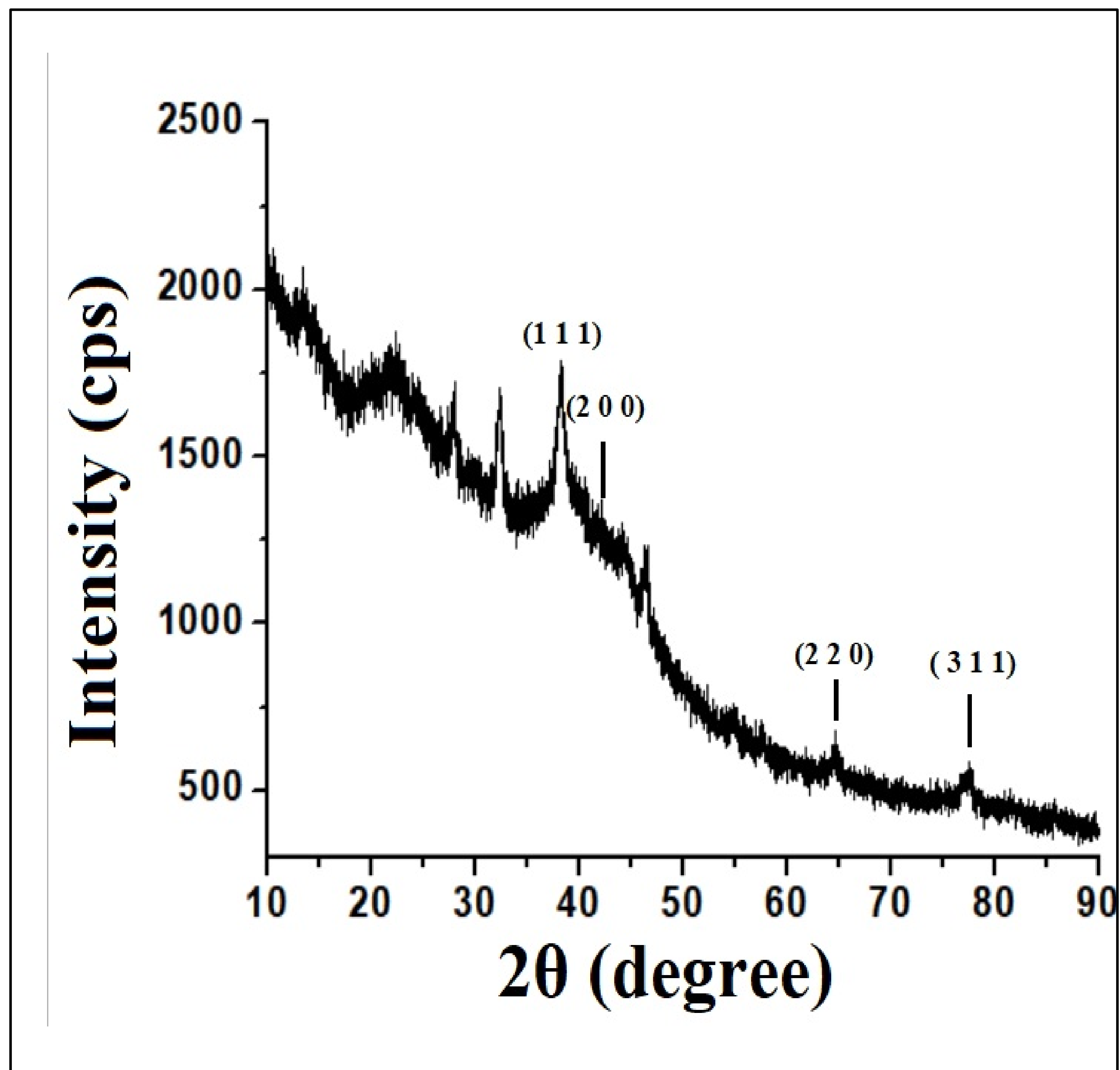

Figure 4. XRD pattern of the Ag-NPs
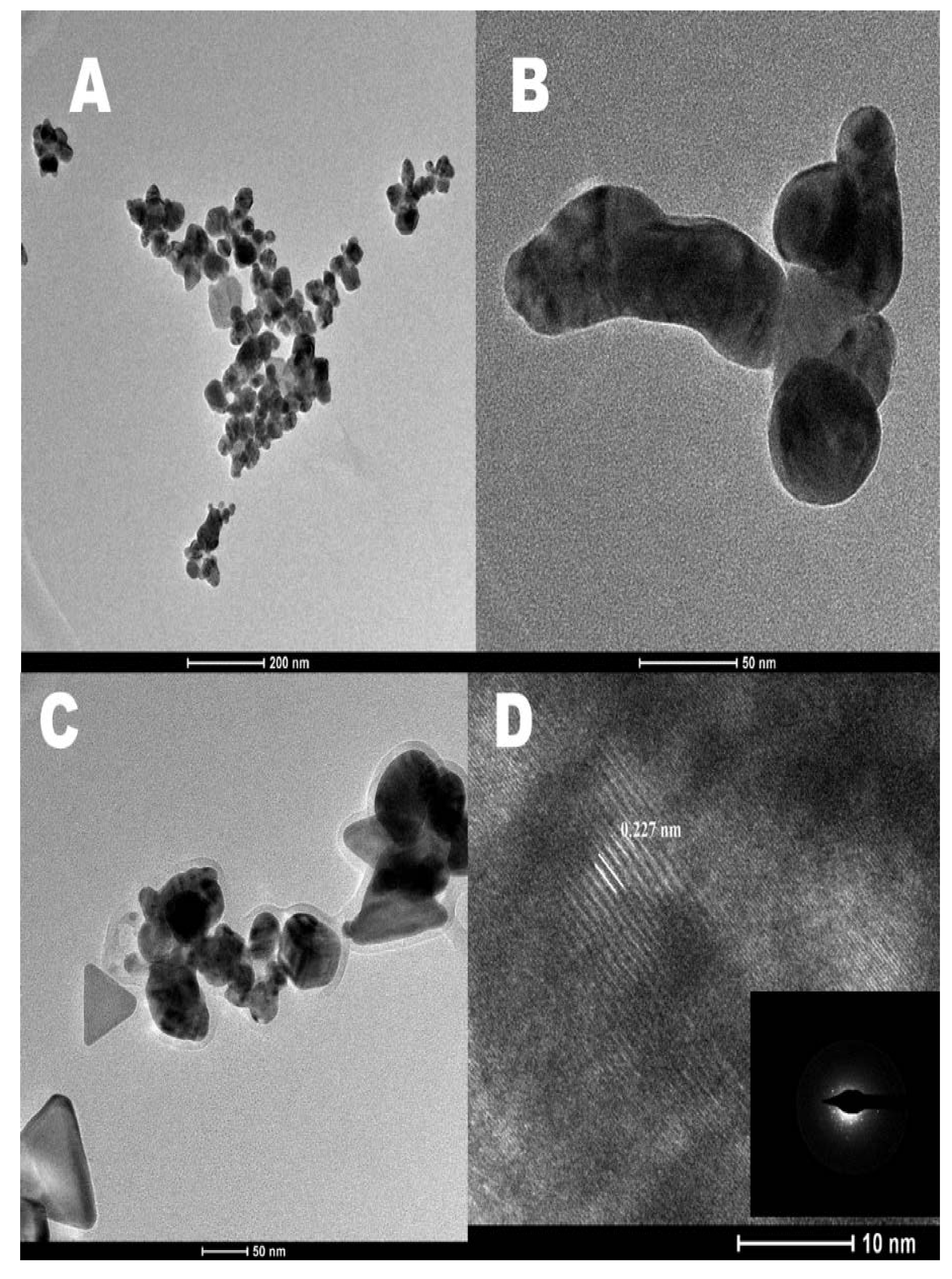

Figure 5: TEM images of alginate-capped silver nanoparticles a (A) $1 \mathrm{hr}$, (B) $24 \mathrm{hr}$ and (C) $48 \mathrm{hr}$. (D) HRTEM image along with the corresponding SAED pattern (inset).

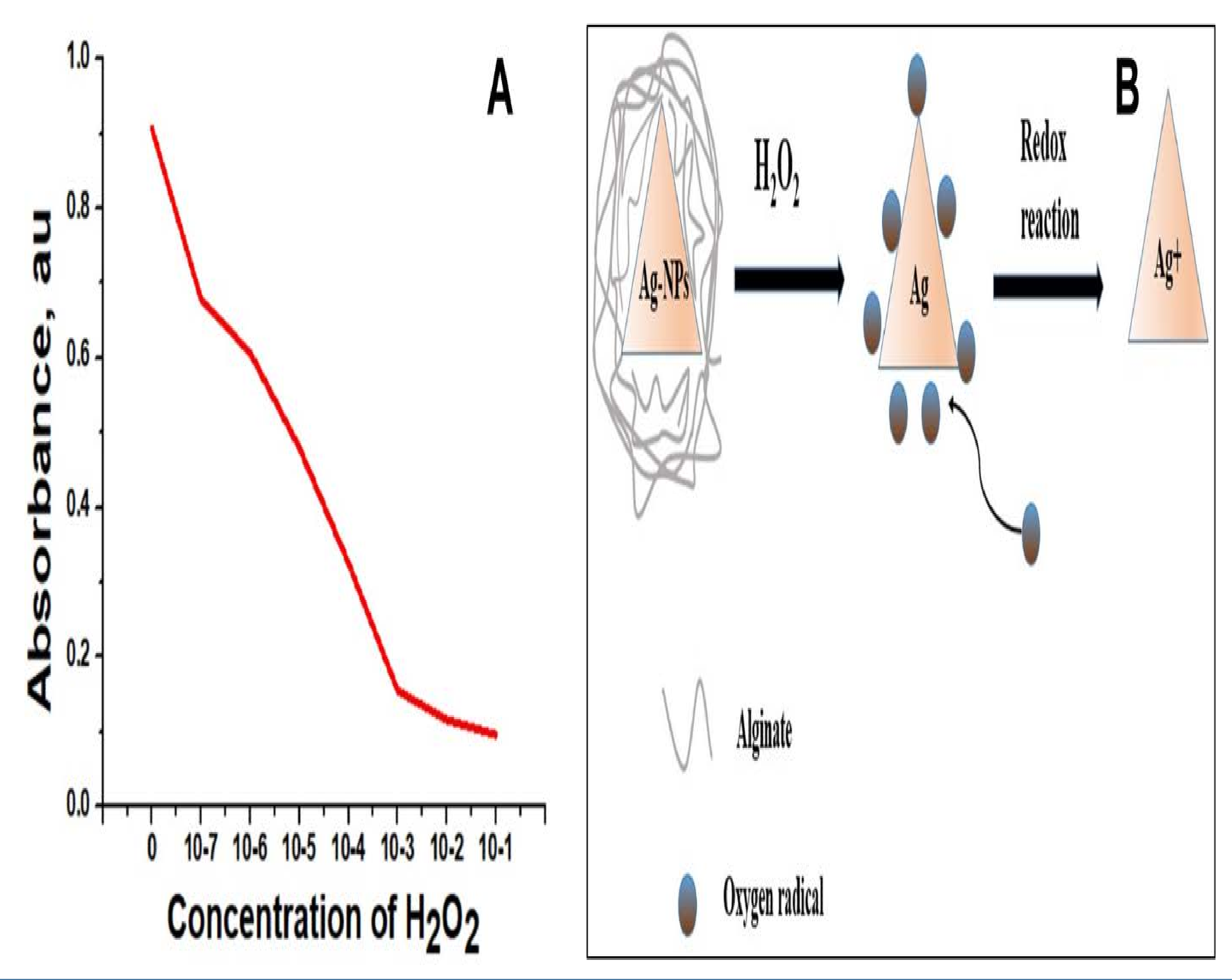

Figure 7: (A) Change in absorbance versus $\mathrm{H} 2 \mathrm{O} 2$ concentration (B) Possible mechanism of the reaction between the Ag-NPs and $\mathrm{H} 2 \mathrm{O} 2$

\section{Conclusions}

An innovative, simple, and green method for the synthesis of stable heterostructured sodium alginate-capped silver nanoparticles is reported. Sodium alginate was used as the capping agent, while glucose acted as the reducing agent. The as-synthesized Ag-NPs were small (30-45 nm) and spherical during the initial hours (1-10) hours of the reaction and evolved to become heterostructured after 48 hours. The as-synthesized Ag-NPs showed excellent sensing activity towards the addition of hydrogen peroxide even at a very low concentration of 10-7 M H2O2.

\section{Acknowledgements}

This project is supported by Qatar University Collaborative Grant QUCG-CAM-19/20-2. The findings achieved herein are solely the responsibility of the authors.

\section{References}

Darroudi, M: Ahmad M. B: Zamiri, R. Zak A K: Abdullah, A H Ibrahim, N. A. Int. J. Nanomedicine 2011, 6, 677-681.7

1. Raveendran, P.; Fu, J.; Wallen, S. L. Completely "green" synthesis and stabilization of metal nanoparticles. J Am. Chem. Soc. 2003,125(46), 13940-13941.

3. S. Mohan, O.S. Oluwafemi, S.C. George, V. P. Jayachandran, F. B. Lewu S. P. Songca, N. Kalarikkal, S. Thomas, Carbohyd. Polym. 106 (2014) 469-474.

4. Pal, T.; Maity, D. S.; Ganguly, A. Talanta, 1988, 35(8), 658-660.

5. T.C. Mokhena, N.V Jacobs, A.S. Luyt; eXPRESS Polym. Lett. 2017 11(8), 652-663 